\title{
Construction of Fully Symmetric Numerical Integration Formulas
}

\author{
J. McNamee and F. Stenger
}

Received September 26, 1966

\begin{abstract}
The paper develops a construction for finding fully symmetric integration formulas of arbitrary degree $2 k+1$ in $n$-space such that the number of evaluation points is $O\left((2 n)^{k} / k !\right), n \rightarrow \infty$. Formulas of degrees $3,5,7,9$, are relatively simple and are presented in detail. The method has been tested by obtaining some special formulas of degrees 7,9 and 11 but these are not presented here.
\end{abstract}

\section{Introduction}

The practical problem in approximation of $n$-dimensional integrals is to find a method of evaluation which can be used on a computer, yields a desired accuracy and is not unduly time-consuming. No attempt is made here to present even a skeleton review of the methods which have been explored. The paper is confined to methods which depend on the evaluation of the integrand at a predetermined set of points; some previous results of other workers which are germane to the present investigation are outlined briefly.

Some outstanding difficulties in the field of $n$-dimensional quadrature formulas are the following:

1. In general, the choice of points will depend on the domain of integration.

2. The choice of points involves the solution of non-linear simultaneous equations.

3. In general, the number of points needed will be an increasing function of $n$, the dimension of the domain of integration.

4. For practical reasons, formulas with positive weights are to be preferred.

5. Practical error bounds are needed.

We consider these difficulties in order. The first four difficulties can be evaded to some extent, following the lead of Thacher, Hammer, Wymore, and Stroud, who directed attention to tractable problems in a series of papers, $[1-3]$ and $[4]$. First, we may seek a transformation of a given domain into a domain of simpler geometry as in Section 2 of the present paper. Second, we may seek formulas applicable to symmetric regions and it then turns out that the number of nonlinear equations is independent of $n$. The kind of symmetry envisaged is explained in Section 3. An explicit construction of special fully symmetric integration formulas of degree 3, 5, 7 and 9 is carried out in Section 4 by solution of non-linear algebraic equations. Difficulties encountered in Section 4 are removed in Sections 5,6 and 7 ; the developments of these latter sections and of Section 3 
culminate in the constructive proof of Section 8. A general construction for fully symmetric formulas of arbitrary degree $2 k+1$ requiring the least possible number of evaluation points, viz.

is given in Section 8 .

$$
\frac{(2 n)^{k}}{k !}\left[1+O\left(\frac{1}{n}\right)\right] \quad(n \rightarrow \infty)
$$

The formulas obtained may be considered as a subset of a general class envisaged by LYNESS [5-7], though our procedure is simple and direct in that we have introduced relatively few definitions and we have reduced the problem to a system of linear algebraic equations. All minimum-point fully symmetric quadrature formulas have the unfortunate property that some of the weights become very large and negative for large $n$. This reduces the accuracy of the formulas and also leads to a gross over-estimate in some methods of bounding the error of quadrature. TсhakalofF [9] has given an interesting result in this direction. He showed that a positive weight formula can always be found for an $n$-dimensional domain and that the evaluation points are interior to the domain. Unfortunately his result presupposes $\left(\begin{array}{c}n+k \\ k\end{array}\right)$ evaluation points and this is impractical even for moderate $n$ and $k$. Precise results on the least number of points in positive weight formulas are much needed.

Error bounds are omitted in the present paper. The few known results applicable to fully symmetric integration formulas are not of practical value. Some new results for repeated Gauss-type quadrature formulas have been obtained recently [10] and it is hoped that analogous results can be obtained for the fully symmetric formulas described in this paper.

\section{Transformation from an Arbitrary Region to the $\boldsymbol{n}$-Cube}

Let $\boldsymbol{R}^{n}$ and $\boldsymbol{S}^{n}$ be regions in Euclidean $n$-space $\boldsymbol{E}^{n}$. HAMmer and WyMORE proved a theorem in [2] which enables one to apply a known integration formula over $\boldsymbol{R}^{n}$ to $\boldsymbol{S}^{n}$ whenever there exists a transformation with continuous nonvanishing jacobian which transforms $\boldsymbol{R}^{n}$ onto $\boldsymbol{S}^{n}$. Although the value of this theorem is indisputable, since the transformation from $\boldsymbol{R}^{n}$ to $\boldsymbol{S}^{n}$ is not in general known, the theorem is not in most suitable form from the point of view of applications.

We prefer to reverse the procedure of HAMmER and WyMORE, in that we shall explicitly construct a transformation from a class of regions $\left\{\boldsymbol{S}^{n}\right\}$ to a particular region $\boldsymbol{R}^{n}$. The procedure is given in the proof of the following theorem.

Theorem 2.1. The integral

$$
\begin{aligned}
& I=\int_{\boldsymbol{g}_{1}}^{h_{1}} \int_{\boldsymbol{g}_{2}}^{h_{2}} \cdots \int_{\mathbf{g}_{n}}^{h_{n}} f(x) d V(x) \\
& \boldsymbol{x}=x^{1}, x^{2}, \ldots, x^{n} ; \quad d V(\boldsymbol{x})=d x^{1} d x^{2} \ldots d x^{n}
\end{aligned}
$$

can always be transformed into a integral over the $n$-cube by a sequence of $n$ linear transformations. The functions $h_{i}$ and $g_{i}$ are assumed to be continuous and bounded almost everywhere and depend only on the variables $x^{1}, x^{2}, \ldots, x^{i-1}, g_{1}$ and $h_{1}$ being constant. 
Proof. The symmetric case $g_{i}=-h_{i}$ is easily tractable and we consider it first. We set

$$
x^{i}=h_{i} u^{i}, \quad i=1,2, \ldots, n
$$

Then

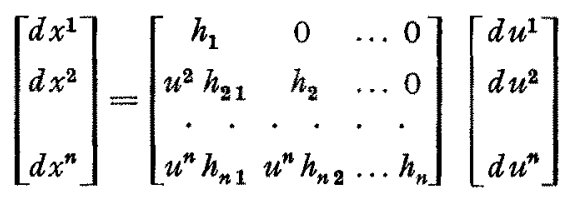

where we have set $h_{i j}=\partial h_{i} / \partial w^{j}$. From (2.3) the jacobian $J$ of the transformation (2.2) is just the product of the diagonal elements of the matrix:

$$
J=\prod_{i=1}^{n} h_{i}
$$

Hence we have

$$
\int_{-h_{3}}^{h_{1}} \cdots \int_{-h_{n}}^{h_{n}} f(\boldsymbol{x}) d V(\boldsymbol{x})=\int_{-1}^{1} \cdots \int_{-1}^{1} F(\boldsymbol{u}) J d V(\boldsymbol{u})
$$

where $J$ is given by (2.4) and $F=f$ under the transformation (2.2).

If the limits of integration are as in (2.1) then the set of transformations

$$
x^{i}=\frac{h_{i}+g_{i}}{2}+u^{i} \frac{h_{i}-g_{i}}{2}, \quad i=1,2, \ldots, n
$$

will transform the integral (2.1) to an integral over the $n$-cube, and the proof is similar to that for the symmetric case above. The jacobian of the transformation (2.6) is

$$
J=\prod_{i=1}^{n}\left(\frac{h_{i}-g_{i}}{2}\right)
$$

As an example, let us transform the integral

$$
I=\int_{-1}^{1} \int_{-\sqrt{1-x^{2}}}^{\sqrt{1-x^{2}}} f(x, y) d y d x
$$

into the corresponding integral over the square. By Eq. (2.2) the linear transformations which will enable us to do this are $x=t, y=s \sqrt{1-x^{2}}=s \sqrt{1-t^{2}}$. By Eq. (2.4) the jacobian of the transformation is $\sqrt{1-t^{2}}$. Hence

$$
I=\int_{-1}^{1} \int_{-1}^{1} f\left(t, s \sqrt{1-t^{2}}\right) \sqrt{1-t^{2}} d s d t
$$

We can now evaluate this integral by e.g. repeated Gaussian integration: GaussLegendre in the variable $x$, Gauss-Chebyshev ${ }^{1}$ in the variable $t$.

Theorem 2.1 is of course limited in scope to transformations of the type (2.6). Within this limitation the theorem is of practical value since it enables one to prescind from the task of finding integration formulas valid for arbitrary regions

$$
1 \int_{-1}^{1} \sqrt{1-x^{2}} f(x) d x \cong \sum_{i=1}^{m} b_{i} f\left(x_{i}\right) ; \quad b_{i}=\frac{\sin ^{2}\left(\frac{j \pi}{m+1}\right)}{m+1}, \quad x_{j}=\cos \left(\frac{j \pi}{m+1}\right) .
$$


(over which the integrals may be written as in (2.1)) in $n$-space and to concentrate on the simpler problem of finding higher-degree integration formulas for the $n$-cube, keeping the weight function arbitrary.

\section{Fully Symmetric Generators}

The following definitions are used.

(a) $A$ set $\boldsymbol{R}^{n}$ in Euclidean $n$-space $\boldsymbol{E}^{n}$ is said to be fully symmetric if $\boldsymbol{x} \in \boldsymbol{R}^{n}$ implies $\boldsymbol{y} \in \boldsymbol{R}^{n}$ where $\boldsymbol{y}$ is any point obtainable from $\boldsymbol{x}$ by permutations and by changes of sign of the coordinates of $\boldsymbol{x}$.

(b) A function $g$ defined on a fully symmetric set is fully symmetric if $g(\boldsymbol{x})=g(\boldsymbol{y})$.

It follows that any fully symmetric set $\boldsymbol{S}$ of a finite number of points can be decomposed into a finite number of disjoint classes $\boldsymbol{S}_{j}(j=1,2, \ldots, m)$ with the property that any member of a particular class can be used to generate the whole class. A particular point

$$
\left(u_{1}, u_{2}, \ldots, u_{r}, 0, \ldots, 0\right)
$$

in $\boldsymbol{E}^{n}$, where $0<u_{i} \leqq u_{j}$ if $i \leqq j$, will be referred to as a generator, and displayed as

$$
\left[u_{1}, u_{2}, \ldots, u_{r}\right] \text {. }
$$

For convenience the zero coordinates have been suppressed.

We shall consider numerical integration formulas of the type

$$
I(f) \equiv \int_{\boldsymbol{R}^{n}} w(\boldsymbol{x}) f(\boldsymbol{x}) d V(\boldsymbol{x}) \cong \sum_{j=1}^{m} w_{j} f\left(\boldsymbol{x}_{j}\right) \equiv J_{m}(f)
$$

where $\boldsymbol{R}^{n}$ and $w(\boldsymbol{x})$ are fully symmetric, and $w(\boldsymbol{x})>0$ in $\boldsymbol{R}^{n}$. The formula (3.3) will be referred to as a fully symmetric numerical integration formula if the set $\boldsymbol{S}$ of evaluation points is a fully symmetric set, and $\mathbf{S}$ is the union of fully symmetric sets $\mathbf{S}_{i}$ generated by distinct generators (i.e. $\mathbf{S}_{i} \cap \mathbf{S}_{j}$ is empty if $i \neq 1$ ) such that to every generator there corresponds exactly one weight ${ }^{2}$.

Let the generator (3.2) have $p$ distinct positive coordinates: $p_{1}$ of the first, $\ldots, p_{p}$ of the $p$-th so that $\sum_{j=1}^{p} p_{j}=r$. Then by rearranging these coordinate values in all possible ways and allowing all possible changes of signs of the coordinates we obtain

$$
N\left(p_{1}, p_{2}, \ldots, p_{p}\right)=\frac{2^{r} n !}{(n-r) ! \prod_{j=1}^{p} p_{j} !}
$$

different points in $n$-space.

Let (3.3) be a fully symmetric integration formula. Then it follows that

(c) If in (3.3) $f$ is a monomial containing an odd power of a coordinate variable then $I(f)=J_{m}(f)=0$.

(d) If $f$ is a monomial containing only even powers of variables then $I(f)$ and $J_{m}(f)$ depend only on the exponents and not on the ordering of the variables.

2 The weights in (3.3) are the numbers $w_{i}$. 
It is clear from the last of the above statements that if a fully symmetric integration formula is exact for all monomials up to some even degree $2 k$, then it is exact for all monomials of degree $2 k+1$.

(e) The fully symmetric integration formula (3.3) is said to be of degree $2 k+1$ whenever it is exact for all monomials of the form

$$
\begin{array}{ll}
\left(x^{1}\right)^{k_{1}}\left(x^{2}\right)^{k_{2}} \ldots\left(x^{n}\right)^{k_{n}} & k_{i} \text { integer } \\
0 \leqq k_{i} \leqq k_{j} \quad \text { if } \quad i \leqq j, & \sum_{j=1}^{n} k_{j} \leqq 2 k+1 \quad \text { (odd integer). }
\end{array}
$$

In constructing fully symmetric integration formulas we may thus restrict ourselves to all the distinct monomials of the form

$$
\begin{aligned}
& \left(x^{1}\right)^{2 k_{1}}\left(x^{2}\right)^{2 k_{1}} \ldots\left(x^{y}\right)^{2 k_{v}} \quad(\equiv 1 \text { if } y=0) \\
& 1 \leqq k_{i} \leqq k_{j} \quad \text { if } \quad i \leqq j, \quad \sum_{j=1}^{y} k_{j} \leqq k
\end{aligned}
$$

where the $k_{i}$ are positive integers.

It is also convenient to use the notation

$$
\sum t\left[u_{1}, u_{2}, \ldots, u_{r}\right]
$$

to imply that the sum extends over all the $N\left(p_{1}, p_{2}, \ldots, p_{p}\right)$ points generated by the generator (3.2).

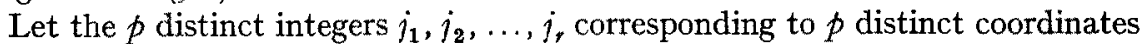
in the generator $[\boldsymbol{G}]=\left[u_{j_{1}}, \ldots, u_{j_{r}}\right]$ range from 1 to $p$. Let $p_{j}$ be the number of times $u_{j}$ appears in $G, j=1,2, \ldots, p$. With $\nu(\nu \leqq r)$ any positive integer we substitute the monomial (3.6) into $\sum f[\boldsymbol{G}]$ to obtain the fully symmetric polynomial

$$
2^{r} \cdot \sum_{i_{1}=1}^{p} \ldots \sum_{i_{v}=1}^{p} \varrho_{r}\left(i_{1}, \ldots, i_{v}\right) u_{i_{1}}^{2 k_{1}} \ldots u_{i_{v}}^{2 k_{v}}
$$

where $Q_{r}\left(i_{1}, \ldots, i_{\nu}\right)$ is defined as follows. Corresponding to the integers $p_{j}$ we define integers $q_{j}$, where $q_{j}$ denotes the number of times the integer $j$ appears in $\left(i_{1}, \ldots, i_{y}\right)$. $\left(q_{j}=0\right.$ if $j$ does not appear in this set.) Then ${ }^{3}$

$$
\varrho_{r}\left(i_{1}, \ldots, i_{v}\right)=\frac{(n-v) !}{(n-v) !}\left[\prod_{j=1}^{p}\left(p_{j}-q_{j}\right) !\right]^{-1},
$$

where we put $\left(p_{j}-q_{j}\right) !=\infty$ if $p_{j}-q_{j}<0$.

The formula (3.4) indicates the manner in which we ought to choose our generators in order to obtain fully symmetric integration formulas which require a minimum number of points. Once the total number of generators to be used is fixed, the following procedure evidently minimizes the number of points generated by each generator:

(f) Minimization of $r$, the total number of non-zero corodinates in a generator; and

(g) Minimization of $p$, the total number of distinct positive coordinates in a generator.

${ }^{3}$ In the evaluation of $\Sigma f[\boldsymbol{G}]$ where $f$ is the monomial (3.6) we observe that the $(n-v)$ zeros in each evaluation point must occupy $(n-v)$ of the $(n-v)$ last coordinate positions. The remaining $(n-v)-(n-n)$ positions are shared by $\left(p_{j}-q_{j}\right) u_{j}$ s $j=1$, $2, \ldots, p$. With $i_{1}, i_{2}, \ldots, i_{n}$ fixed, the number of distinct possibilities therefore is $\varrho_{r}\left(i_{1}, \ldots, i_{v}\right)$ given by $(3.9)$. 


\section{Construction of Fully Symmetric Formulas of Degree 3, 5, 7 and 9}

In this section we shall write down the non-linear algebraic equations and their corresponding solutions, which yield fully symmetric integration formulas of degree $3,5,7$ and 9 . It is convenient to use the notations

$$
\begin{aligned}
& n^{(k)}=n(n-1) \ldots(n-k+1) \\
& n_{(k)}=\frac{n(n-1) \ldots(n-k+1)}{k !} .
\end{aligned}
$$

The following generators suffice for these formulas.

$\begin{array}{ll}\text { Generator } & \text { Number of points } \\ {[0]} & 1 \\ {[u]} & 2 n \\ {[u, u]} & 2 n^{(2)} \\ {[u, v]} & 2^{2} n^{(2)} \\ {[u, u, u]} & 2^{3} n_{(3)} \\ {[u, u, u, u]} & 2^{4} n_{(4)}\end{array}$

On the right side of the non-linear algebraic equations we designate

$$
\int_{\boldsymbol{R}^{n}} w(\boldsymbol{x})\left(x^{i}\right)^{2}\left(x^{j}\right)^{4} d V(\boldsymbol{x}), \quad i \neq j
$$

for example by $I_{2,4}\left(=I_{4,2}\right)$.

\subsection{Formulas of Degree 3}

We attempt to obtain the following formula of degree 3

$$
I(f) \cong A_{0} f[0]+A_{1} \sum t[u] \text {. }
$$

The non-linear equations to be satisfied are

$$
\left(\begin{array}{ll}
1 & 2 n \\
0 & 2 u^{2}
\end{array}\right)\left(\begin{array}{l}
A_{0} \\
A_{1}
\end{array}\right)=\left(\begin{array}{l}
I_{0} \\
I_{2}
\end{array}\right)
$$

Clearly (4.3) is satisfied if we first choose $u$ and then solve for the linear unknowns, viz.

$$
\begin{aligned}
& u^{2}=I_{2} / I_{0} \\
& A_{1}=I_{0} /\left(2 u^{2}\right), \quad A_{0}=(1-n) I_{0} .
\end{aligned}
$$

\subsection{Formulas of Degree 5}

We attempt a $2 n^{2}+1$-point formula of the form

$$
I(f) \cong A_{0} f[0]+A_{1} \sum f[u]+A_{1,1} \sum f[u, u] .
$$

4 If instead of (4.2) we had chosen

\section{$(4.2)^{\prime}$}

$$
I(f) \cong A \Sigma f[u]
$$

and demanded that the right hand side be exact for any polynomial of degree 3 , we would obtain the unique solution $u=\sqrt{n I_{2} / I_{0}}, A=I_{0} / 2 n$. However $(4.2)^{\prime}$ does not fit our theory to be developed in later sections; moreover the evaluation points $u$ for $(4.2)^{\prime}$ are eventually outside $\boldsymbol{R}^{n}$ for $n$ sufficiently large. It may be noted that (4.2)' has positive weights. It will be seen later that adherence to positive weight formulas can lead to other inconvenient properties, e.g. evaluation points outside the domain. or complex evaluation points. 
Using (3.8) we attempt to satisfy the following system of non-linear algebraic equations.

$$
\left(\begin{array}{lll}
1 & 2 n & 2 n^{(2)} \\
0 & 2 u^{2} & 4(n-1) u^{2} \\
0 & 2 u^{4} & 4(n-1) u^{4} \\
0 & 0 & 4 u^{4}
\end{array}\right)\left(\begin{array}{l}
A_{0} \\
A_{1} \\
A_{1,1}
\end{array}\right)=\left(\begin{array}{l}
I_{0} \\
I_{2} \\
I_{4} \\
I_{2,2}
\end{array}\right)
$$

The value of $u$ is easily obtained by dividing the second equation into the third. By ordinary elimination we can then obtain the value of the linear coefficients. The general solution is

$$
\begin{aligned}
u & =\left(I_{4} / I_{2}\right)^{\frac{1}{2}} \\
A_{0} & =I_{0}-n\left(\frac{I_{2}}{I_{4}}\right)^{2}\left[I_{4}-\frac{n-1}{2} I_{2,2}\right] \\
A_{1} & =\frac{1}{2}\left(\frac{I_{2}}{I_{4}}\right)^{2}\left[I_{4}-(n-1) I_{2,2}\right] ; \quad A_{1,1}=\frac{1}{4}\left(\frac{I_{2}}{I_{4}}\right)^{2} I_{2,2},
\end{aligned}
$$

\subsection{Formulas of Degree 7}

We attempt to obtain a formula of degree 7 of the form

$$
\begin{aligned}
I(t) \cong & A_{0} f[0]+A_{1} \sum f[u]+A_{2} \sum f[v] \\
& +A_{1,1} \sum f[u, u]+A_{2,2} \sum f[v, v] \\
& +A_{1,1,1} \sum f[u, u, u] .
\end{aligned}
$$

To obtain the value of $u, v, A_{0}, \ldots, A_{1,1,1}$ in (4.8) we need to solve the following system of non-linear algebraic equations

$$
\left[\begin{array}{llllll}
1 & 2 n & 2 n & 2^{2} n_{(2)} & 2^{2} n_{(2)} & 2^{3} n_{(3)} \\
0 & 2 u^{2} & 2 v^{2} & 2^{2}(n-1) u^{2} & 2^{2}(n-1) v^{2} & 2^{3}(n-1)_{(2)} u^{2} \\
0 & 2 u^{4} & 2 v^{4} & 2^{2}(n-1) u^{4} & 2^{2}(n-1) v^{4} & 2^{3}(n-1)_{(2)} u^{4} \\
0 & 2 u^{6} & 2 v^{6} & 2^{2}(n-1) u^{6} & 2^{2}(n-1) v^{6} & 2^{3}(n-1)_{(2)} u^{6} \\
0 & 0 & 0 & 2^{2} u^{4} & 2^{2} v^{4} & 2^{3}(n-2) u^{4} \\
0 & 0 & 0 & 2^{2} u^{6} & 2^{2} v^{6} & 2^{3}(n-2) u^{6} \\
0 & 0 & 0 & 0 & 0 & 2^{3} u^{6}
\end{array}\right]\left[\begin{array}{l}
A_{0} \\
A_{1} \\
A_{2} \\
A_{1,1} \\
A_{2,2} \\
A_{1,1,1}
\end{array}\right]=\left[\begin{array}{l}
I_{0} \\
I_{2} \\
I_{4} \\
I_{6} \\
I_{2,2} \\
I_{2,4} \\
I_{2,2,2}
\end{array}\right]
$$

(4.9) See Fig. 1.

Fig. 1. The System (4.9)

The solution to the system (4.9) is not unique. Among an infinity ${ }^{5}$ of possible choices of $u$ and $v$ we choose $\pm u$ und $\pm v$ as solutions of

$$
\left(I_{2}^{2}-I_{0} I_{4}\right) u^{4}-\left(I_{2} I_{4}-I_{0} I_{6}\right) u^{2}+\left(I_{4}^{2}-I_{2} I_{6}\right)=0 \text {. }
$$

s $u$ and $v$ need merely be related by $v^{2}=\left(u^{2} I_{4}-I_{6}\right) /\left(u^{2} I_{2}-I_{4}\right)$ in order that the second, third and fourth equations in (4.9) be simultaneously satisfied. Thus we could for example set $A_{1,1}=0$ in (4.8), if we were to define $u$ and $v$ as the zeros of the polynomial

$$
\begin{aligned}
u^{*}\left(I_{2,2} I_{4}-I_{2,4} I_{2}\right) & +u^{4}\left(m I_{2} I_{2,2,2}+I_{4} I_{2,4}-I_{2,2} I_{0}\right) \\
& -2 m u^{8}\left(I_{4} I_{2,2,2}\right)+\left(m I_{6} I_{2,2,2}\right)
\end{aligned}
$$

where $m=n-2$. For all integrals with "property $\boldsymbol{P}$ " (See Section 6) $I_{2,2} I_{4}-I_{2,4} I_{2}=0$, and for large $n$ the zeros of the polynomial (*) will thus be complex. 
In Section 8 it will be made clear that by this choice of $u$ and $v$ the second, third and fourth equations in (4.9) are simultaneously satisfied. Moreover, if $w(\boldsymbol{x})>0, u \neq v$. The linear unknowns are given by

$$
\begin{aligned}
A_{1,1,1} & =I_{2,2,2} /\left(2 u^{2}\right)^{3} \\
\left(\begin{array}{l}
A_{1,1} \\
A_{2,2}
\end{array}\right) & =\frac{1}{4}\left(\begin{array}{ll}
u^{4} & v^{4} \\
u^{6} & v^{6}
\end{array}\right)^{-1}\left[\left(\begin{array}{l}
I_{2,2} \\
I_{2,4}
\end{array}\right)-2^{3}(n-2)\left(\begin{array}{l}
u^{4} \\
u^{6}
\end{array}\right) A_{1,1,1}\right] \\
\left(\begin{array}{l}
A_{1} \\
A_{2}
\end{array}\right) & =-2(n-1)\left(\begin{array}{l}
A_{1,1} \\
A_{2,2}
\end{array}\right)+\frac{1}{2}\left(\begin{array}{ll}
u^{2} & v^{2} \\
u^{4} & v^{4}
\end{array}\right)^{-1}\left[\left(\begin{array}{l}
I_{2} \\
I_{4}
\end{array}\right)-2^{3}(n-1)_{(2)}\left(\begin{array}{l}
u^{2} \\
u_{4}
\end{array}\right) A_{1,1,1}\right. \\
A_{0} & =I_{0}-2 n\left(A_{1}+A_{2}\right)-2^{2} n_{(2)}\left(A_{1,1}+A_{2,2}\right)-2^{3} n_{(3)} A_{1,1,1} .
\end{aligned}
$$

Fig. 2. The Eqs. (4.11)

(4.11) See Fig. 2.

Observe that we obtain two integration formulas by interchanging the role of $u$ and $v$ in the Eqs. (4.9).

\subsection{Formulas of Degree 9}

We attempt to find a formula of the form

$$
\begin{aligned}
I(f) \cong & A_{0} f[0]+A_{1} \sum f[u]+A_{2} \sum f[v] \\
& +A_{1,1} \sum f[u, u]+A_{1,2} \sum f[u, v]+A_{2,2} \sum f[v, v] \\
& +A_{1,1,1} \sum f[u, u, u]+A_{2,2,2} \sum f[v, v, v] \\
& +A_{1,1,1,1} \sum f[u, u, u, u] .
\end{aligned}
$$

The requirement that (4.12) be exact for all polynomials of degree 9 leads to the following system of non-linear equations (see p.335).

(4.13) See Fig. 3.

The solution to (4.13) given below is valid provided

$$
I_{6,2}-\left(u^{2}+v^{2}\right) I_{4,2}+u^{2} v^{2} I_{2,2}=0
$$

or, what is the same thing, provided that

$$
\int_{\boldsymbol{R}^{n}} w(x)\left(x^{i} x^{j}\right)^{2}\left[\left(x^{i}\right)^{2}-u^{2}\right]\left[\left(x^{i}\right)^{2}-v^{2}\right] d V(x)=0 \quad(i \neq j) .
$$

The unknowns $u$ and $v$ are taken to be the zeros of the polynomial

$$
\left(I_{4}^{2}-I_{2} I_{6}\right) u^{4}-\left(I_{4} I_{6}-I_{2} I_{8}\right) u^{2}+I_{6}^{2}-I_{4} I_{8}
$$




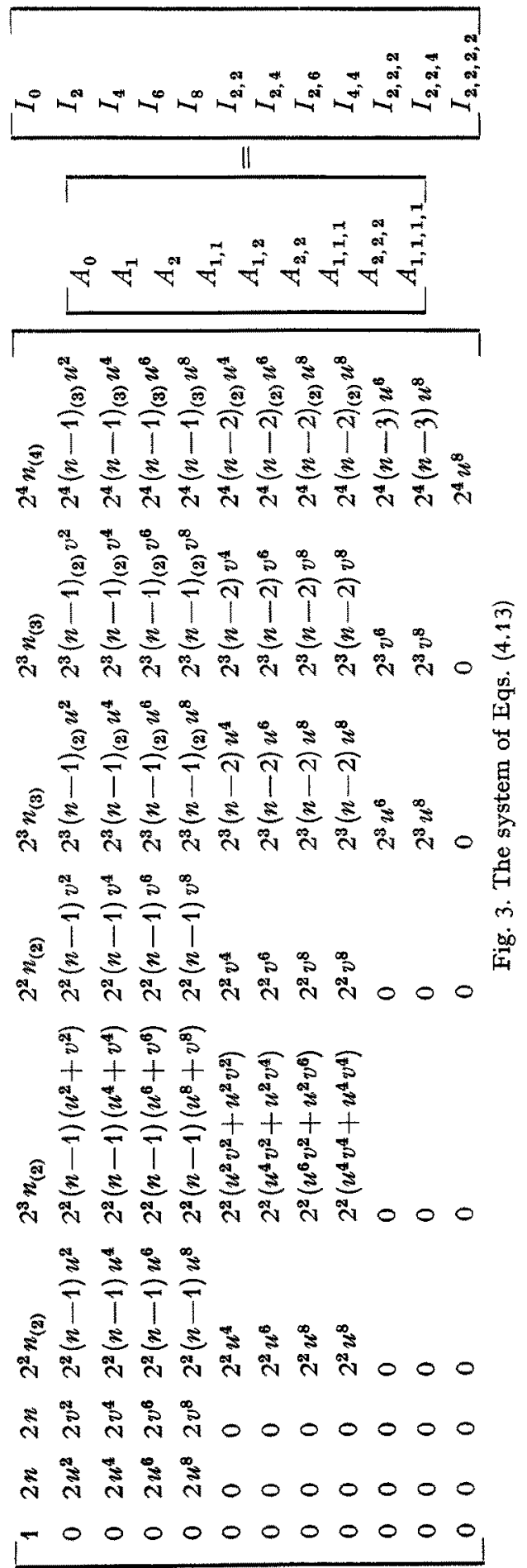


The linear unknowns in (4.13) are then given by

$$
\begin{aligned}
A_{1,1,1,1}= & I_{2,2,2,2} /\left(2 u^{2}\right)^{4} \\
\left(\begin{array}{l}
A_{1,1,1} \\
A_{2,2,2}
\end{array}\right)= & \frac{1}{2^{3}}\left(\begin{array}{ll}
u^{6} & v^{6} \\
u^{8} & v^{8}
\end{array}\right)^{-1}\left[\left(\begin{array}{l}
I_{2,2,2} \\
I_{2,2,4}
\end{array}\right)-2^{4}(n-3) A_{1,1,1,1}\left(\begin{array}{l}
u^{6} \\
u^{8}
\end{array}\right)\right] \\
A_{1,2}= & \left(I_{2,8}-I_{4,4}\right) /\left[2^{2} u^{2} v^{2}\left(u^{2}-v^{2}\right)^{2}\right] \\
\left(\begin{array}{l}
A_{1,1} \\
A_{2,2}
\end{array}\right)= & -2(n-2)\left(\begin{array}{l}
A_{1,1,1} \\
A_{2,2,2}
\end{array}\right)+\frac{1}{2^{2}}\left(\begin{array}{ll}
u^{6} & v^{8} \\
u^{8} & v^{8}
\end{array}\right)^{-1}\left[\left(\begin{array}{l}
I_{2,4} \\
I_{2,6}
\end{array}\right)\right. \\
& \left.-2^{2}\left(\begin{array}{l}
u^{4} v^{2}+u^{2} v^{4} \\
u^{6} v^{2}+u^{2} v^{8}
\end{array}\right) A_{1,2}-2^{4}(n-2)_{(2)}\left(\begin{array}{l}
u^{6} \\
u^{8}
\end{array}\right) A_{1,1,1,1}\right] \\
\left(\begin{array}{l}
A_{1} \\
A_{2}
\end{array}\right)= & -2(n-1)\left(\begin{array}{l}
A_{1,1}+A_{1,2} \\
A_{2,2}+A_{1,2}
\end{array}\right)-2^{2}(n-1)_{(2)}\left(\begin{array}{l}
A_{1,1,1} \\
A_{2,2,2}
\end{array}\right) \\
& +\frac{1}{2}\left(\begin{array}{ll}
u^{2} & v^{2} \\
u^{4} & v^{4}
\end{array}\right)^{-1}\left[\left(\begin{array}{l}
I_{2} \\
I_{4}
\end{array}\right)-2^{4}(n-1)_{(3)}\left(\begin{array}{l}
u^{2} \\
u^{4}
\end{array}\right) A_{1,1,1,1}\right] \\
A_{0}= & I_{0}-2 n\left(A_{1}+A_{2}\right)-2^{2} n_{(2)}\left(A_{1,1}+2 A_{1,2}+A_{2,2}\right) \\
& -2^{3} n_{(3)}\left(A_{1,1,1}+A_{2,2,2}\right)-2^{4} n_{(4)} A_{1,1,1,1}
\end{aligned}
$$

Fig. 4. The Eqs. (4.17)

\section{(4.17) See Fig. 4.}

By interchanging the role of $u$ and $v$ in (4.13) we obtain two formulas of degree 9.

It will be shown in Section 8 that although the 4-th, 5-th, and 8-th equation in (4.13) have not been used to compute the linear unknowns, these equations are automatically satisfied by our choice of $u$ and $v$ in (4.16).

\section{Orthogonal Polynomials}

The integration formulas we have obtained in Section 4 and those we shall construct in Section 8 have the property that the coordinates in the evaluation points are zeros of polynomials orthogonal over $\boldsymbol{R}^{n}$ with respect to $w(\boldsymbol{x})$. These polynomials $q_{k}(x)\left(q_{k}(x)\right.$ of degree $k$ in $\left.x\right)$ are defined, apart from an arbitrary multiplicative constant by $q_{0}=1$,

$$
\int_{\boldsymbol{n}^{n}} w(\boldsymbol{x}) q_{k}\left(x^{i}\right)\left(x^{i}\right)^{i} d V(\boldsymbol{x})=0, \quad j=0,1, \ldots, k-1, \quad k=1,2,3, \ldots
$$

It is readily seen that the non-linear unknowns used to obtain formulas of degree $3,5,7$ and 9 are the zeros of the polynomials $q_{2}\left(x^{i}\right), q_{3}\left(x^{i}\right), q_{4}\left(x^{i}\right)$ and $q_{5}\left(x^{i}\right)$ respectively.

Under the assumption that $w(\boldsymbol{x})>0$ for $\boldsymbol{x} \in \boldsymbol{R}^{n}$, the zeros of the polynomials $q_{k}\left(x^{i}\right)$ are easily shown to be real and distinct. Moreover, $q_{k}\left(x^{i}\right)$ is either an even or an odd function of $x^{i}$ depending on $k$, and hence [k/2] of the zeros of $q_{k}\left(x^{i}\right)$ will be positive and $[k / 2]$ negative. 


\section{A Restriction on the Integrals}

Although the formulas of degree 3,5 and 7 obtained in Section 4 are valid for arbitrary fully symmetric integrals, the Eq. (4.15) does not hold for all such integrals. It is therefore convenient to introduce the following definition.

Definition 6.1. Let $\boldsymbol{R}^{n}$ and w be fully symmetric, and let the integrals

$$
\int_{\boldsymbol{R}^{n}}^{n} w(\boldsymbol{x}) \prod_{i=1}^{n}\left(x^{i}\right)^{k_{i}} d V(\boldsymbol{x}), \quad k_{i}=0,1,2, \ldots
$$

exist. The integral $I(f)$ on the left of (3.3) will be said to possess property $\boldsymbol{P}$ if there exists a transformation $\boldsymbol{T}, \boldsymbol{T} \boldsymbol{x}=\boldsymbol{y}$ with continuous non-vanishing jacobian which transforms (6.1) into

$$
\int_{-a}^{a} w_{1}\left(y^{1} ; k_{1}\right) d y^{1} \int_{\boldsymbol{R}^{n-1}} w_{n-1}\left(y^{2}, y^{3}, \ldots, y^{n} ; k_{2}, k_{3}, \ldots, k_{n}\right) d V\left(\boldsymbol{y}_{n-1}\right)
$$

where $w_{n-1}$ and $\boldsymbol{R}^{n-1}$ need not be fully symmetric ${ }^{6}$.

With $u$ and $v$ zeros of $q_{5}(x)$, the Eq. (4.15) holds for all integrals with property $\boldsymbol{P}$. Moreover we have

Lemma 6.1. Let the integral (6.1) have property $\boldsymbol{P}$. Then

$$
I_{2 k_{k}, 2 k_{1}, \ldots, 2 k_{r}}=\alpha_{r} I_{2 k_{1}} I_{2 k_{s}} \ldots I_{2 k_{r}}
$$

where $\alpha_{1}=1$ and $\alpha_{r}>0$ is independent of the integers $k_{1}, k_{2}, \ldots, k_{r}$.

Proof. By (6.1) and (6.2) we have

$$
I_{2 k_{1}, 2 k_{\mathrm{g}}}=A\left(2 k_{1}\right) I_{2 k_{\mathrm{z}}}=A\left(2 k_{2}\right) I_{2 k_{1}}
$$

where $A(\cdot)$ is a unique function. Clearly (6.3) follows from (6.4) for $r=2$. The general case follows similarly by induction.

\section{Repeated Integration and Generators}

Assume that we are given an interval $(a, b)(0<a<b)$ and $m$ distinct points $u_{1}, u_{2}, \ldots, u_{m}$ in $(a, b)$. Define

$$
I_{k i}^{(i)}=\int_{a}^{b} w_{i}\left(x^{i}\right)\left(x^{i}\right)^{k_{s}} d x^{i}, \quad k_{i}=1,2,3, \ldots
$$

We can then clearly choose $n$ formulas

$$
\sum_{j=1}^{m} w(i) u_{i}^{k_{i}}=I_{k s}^{(i)}, \quad k_{i}=1,2, \ldots, m ; \quad i=1,2, \ldots, n .
$$

Using (7.2) we can construct the repeated integration formula

$$
\int_{a}^{b} \ldots \int_{a}^{b} f\left(x^{1}, \ldots, x^{n}\right) \prod_{i=1}^{n} w_{i}\left(x^{i}\right) d x^{i} \cong \sum_{j_{1}=1}^{m} \ldots \sum_{j_{n}=1}^{m} w w_{j_{1}}^{(1)} \ldots w_{j_{n}}^{(n)} f\left(u_{j_{1}}, \ldots, u_{j_{n}}\right)
$$

- "Property $P$ " in effect implies that the transformation $T$ above transforms a fully symmetric integral into a repeated integral. 
which is exact for all polynomials $f$ of the form

$$
\prod_{i=1}^{n}\left(x^{i}\right)^{k_{i}}, \quad k_{i}=1,2, \ldots, m
$$

Observe that the determinant of the system (7.2) is a Vandermonde determinant and the $m$ parameters $w_{j}^{(i)}$ are uniquely determined for each $i, i=1,2, \ldots, n$. Hence the Eqs. (7.2) uniquely determine the $m n$ parameters $t_{i}^{(i)}$.

We shall show that we can uniquely determine the integration formula (7.3) without knowing all the $m n$ monomial integrals of the form (7.1), but instead knowing the monomial integrals of chosen $(m-1) n+1$ fully symmetric polynomials. The type of fully symmetric polynomials we shall use are those obtained from (7.4) by summing over all the $n$ ! terms of the form (7.4) with the order of the $k_{i}$ fixed but with the order of the coordinates $x^{i}$ interchanged in all possible ways. Clearly, with $k_{i}$ an integer in the range $1 \leqq k_{i} \leqq m$ we can obtain all the fully symmetric polynomials if we restrict the order of the $k_{i}$ to $k_{i} \leqq k_{i}$ if $i \leqq i$. An easy calculation shows that there are

$$
(m+n-1)_{(n)}
$$

distinct fully symmetric polynomials subject to this restricted range of $k_{i}$.

Integrating only a certain $(m-1) n+1$ of all the fully symmetric polynomials described in the above paragraph we obtain the following system of equations

$$
\begin{aligned}
\prod_{i=1}^{n} I_{1}^{(i)} & =\omega_{t, 0} \quad t=1 \\
\prod_{i=1}^{n} I_{1}^{(i)} \sum_{1 \leqq j_{1}<j_{2}<\cdots<j_{s} \leqq n} \prod_{p=1}^{s} \frac{I_{t}^{(j)}}{I_{1}^{(j p)}} & =\omega_{t, s} \quad s=1,2, \ldots, n, \quad t=2,3, \ldots, m .
\end{aligned}
$$

The Eqs. (7.6) lead to the polynomial equations

$$
\sum_{i=0}^{n}(-1)^{i} \omega_{i, i} \xi^{n-i}=\prod_{i=1}^{n}\left(I_{1}^{(i)} \xi-I_{t}^{(i)}\right)=0 \quad t=\omega, 2, \ldots, m .
$$

That is, the Eqs. (7.6) are satisfied if and only if the zeros of the polynomial on the left of $(7.7)$ are the ratios $I_{t}^{(i)} \mid I_{1}^{(i)}$. Thus, for each fixed $i$ the $(m-1) n+1$ equations (7.6) determine every $I_{i}^{(i)}$, apart from a multiplicative constant. The lack of uniqueness is due to the fact that e.g.

$$
\begin{gathered}
\int_{a}^{b} \int_{a}^{b} w_{1}(x) w_{2}(y) f(x) g(y) d x d y \\
=\int_{a}^{b} w_{1}(x) f(x) d x \int_{a}^{b} w_{2}(y) g(y) d y \\
=c \int_{a}^{b} w_{1}(x) f(x) d x \cdot \frac{1}{c} \int_{a}^{b} w_{2}(y) g(y) d y
\end{gathered}
$$

for an arbitrary non-zero constant $c$, i.e. we cannot factor the integral (7.8) in a unique manner. We observe however, that the $(m-1) n+1$ equations (7.6) are 
an independent set, and they uniquely determine every product $\prod_{i=1}^{n} w_{3 i}^{(i)}$ in $(7.3)$, since they uniquely determine every product on the left of the equation

$$
\prod_{i=1}^{n} I_{k_{i}}^{(i)}=\sum_{j_{1}=1}^{m} \cdots \sum_{i_{n}=1}^{m} w_{j_{1}}^{(1)} \ldots w_{j_{n}}^{(n)} u_{j_{1}}^{k_{1}} \ldots u_{j_{n}}^{k_{n}}, \quad k_{1}=1,2, \ldots, m
$$

Two additional observations are of interest. Although $m n$ equations of the form (7.2) completely determine the formula $(7.3),(7.3)$ is exact for $m^{n}$ monomial integrals of the form (7.4). Similarly, though the $(m-1) n+1$ equations (7.6) completely determine the formula $(7.3),(7.3)$ holds for $(m+n-1)_{(n)}$ fully symmetric monomial integrals.

The above remarks lead us to consider determination of the products $w_{j_{1}}^{(1)} w_{k_{2}}^{(2)}$ $\ldots w_{i n}^{(n)}$ directly by use of generators. Let us denote the monomial (7.4) subject to the constraint $k_{i} \leqq k_{j}$ if $i \leqq j$ by $\pi=\pi\left(x^{1}, x^{2}, \ldots, x^{n}\right)$, and let $f=f\left(x^{1}, x^{2}, \ldots, x^{n}\right)$ be the fully symmetric polynomial generated from $\pi$ in the manner described above. Then we have

$$
\begin{aligned}
& \int_{a}^{b} \cdots \int_{a}^{b} f\left(x^{1}, \ldots, x^{n}\right) \prod_{i=1}^{n} w_{i}\left(x^{i}\right) d x^{i}=\sum_{j_{1}=1}^{m} \cdots \sum_{j_{n}=1}^{m} w_{j_{1}}^{(1)} \ldots w_{j_{n}}^{(n)} f\left(u_{j_{1}}, \ldots, u_{j_{n}}\right) \\
& =\sum_{1 \leqq j_{1} \leqq j_{n} \leqq \cdots \leqq j_{n} \leqq m} A_{j_{1}, j_{*}, \ldots, j_{n}}^{*} w_{j_{1}}^{(1)} w_{j_{2}}^{(2)} \ldots w_{j_{n}}^{(n)} \sum^{*} \pi\left[u_{j_{1}}, u_{j_{2}}, \ldots, u_{j_{n}}\right] \\
& =\sum_{1 \leqq j_{1} \leqq j_{n} \leqq \ldots \leqq j_{n} \leqq m} A_{i_{1}, i_{2}}, \ldots, i_{n} f\left(u_{j_{1}}, u_{j_{1}}, \ldots, u_{j_{n}}\right)
\end{aligned}
$$

where $\sum^{*}$ indicates a sum taken over all the points $\left(u_{i_{1}}, u_{i_{2}}, \ldots, u_{i_{n}}\right)$ obtainable from $\left(u_{j_{1}}, u_{j_{2}}, \ldots, u_{j_{n}}\right)$ by interchanging coordinates. In $(7.10) f$ is arbitrary and every constant $A_{j_{1}, j_{z}, \ldots, j_{n}}$ is uniquely determined.

We next index all the generators $\left[u_{i_{1}}, u_{j_{n}}, \ldots, u_{j_{n}}\right]\left(j_{1} \leqq j_{2} \leqq \cdots \leqq j_{n}\right)$ from 1 to $(m+n-1)_{(n)}$ and we also index all the fully symmetric polynomials obtained as described above, from 1 to $(m+n-1)_{(n)}$. The Eqs. $(7.10)$ can then be written in matrix form

$$
\boldsymbol{V} \boldsymbol{W}=\boldsymbol{J}_{k_{2}, k_{3}, \ldots, k_{n}} .
$$

Here $V$ is an $(m+n-1)_{(n)} \times(m+n-1)_{(n)}$ matrix with $(i, \eta)$-th element equal to the $i-t h$ fuly symmetric polynomial evaluated at the $j$-th generator. $\boldsymbol{W}$ is an $(m+n-1)_{(n)} \times 1$ vector whose $j$-th element is the coefficient $A_{j_{1}, i_{2}, \ldots, i_{n}}$ of the generator point with coordinates $\left(u_{j_{1}}, u_{j_{z}}, \ldots, u_{j_{n}}\right)$, and $\boldsymbol{J}_{k_{1}, k_{n}, \ldots, k_{n}}$ is an $(m+n-1)_{(n)} \times 1$ vector whose $i$-th element is the integral of the $i$-th fully symmetric polynomial.

It has already been observed that the coefficients $A_{j_{1}, j_{2}, \ldots, i_{n}}$ in $(7.10)$ are uniquely determined. We have thus proved

Theorem 7.1. The matrix $\boldsymbol{V}$ described above is non-singular.

\section{Minimum-Point Fully Symmetric Formulas of Arbitrary Degree $2 \boldsymbol{k}+\boldsymbol{1}$}

The developments of the previous sections enable us to give a constructive proof of the following theorem.

Theorem 8.1. Let $w(x)$ satisfy the conditions

a) $w(\boldsymbol{x})>0$ for $\boldsymbol{x} \in \boldsymbol{R}^{n}$;

b) $w(\boldsymbol{x})$ is fully symmetric. 
Let the integral $I(f)$ in (8.1) have property $\boldsymbol{P}$. Then there exist fully symmetric numerical integration formulas in $n$ space of the form

$$
\begin{aligned}
I(f) & \equiv \int_{\boldsymbol{R}^{n}} w(x) f(x) d V(\boldsymbol{x}) \\
& \cong A_{0} f[0]+\sum_{s=1}^{k} \sum_{1 \leq i_{1} \leqq i_{s} \leqq \ldots \leqq j_{k}} A_{j_{1}, j_{k}, \ldots, i_{s}} \sum f\left[u_{i_{1}}, u_{j_{2}}, \ldots, u_{j_{k}}\right]
\end{aligned}
$$

which are exact for all monomials of degree $2 k+1, k>0$ an integer. The coordinates of the evaluation points for the formulas on the right of (8.1) are restricted to be the zeros of the polynomials $q_{k+1}(x)$ defined in Section 5. Subject to this restriction the total number of points $m$ required by the formulas (8.1) is minimal

$$
\left(m=\frac{(2 n)^{k}}{k !}\left[1+O\left(\frac{1}{n}\right)\right], n \rightarrow \infty\right) .
$$

Each of the weights $A_{0}, A_{j_{1}, \ldots, j_{s}}$ is real.

A few remarks concerning the manner in which we shall carry out the proof are in order. At the outset of the proof we shall characterize the minimum number of monomials necessary to solve for all the linear unknowns in (8.1), and at the same time establish an order among these monomials. A suitable ordering is also established for the generators, which are selected according to the rules (f) and $(\mathrm{g})$ of Section 3. This brings about a "triangular structure" of the system of non-linear equations. This "triangular structure" enables us to use the analysis of Sections 6 and 7 to carry out a proof by induction that this system of nonlinear equations possesses a unique solution.

Proof of Theorem 8.1. Let us first conveniently subdivide all the monomial integrals. We denote $I(1)$ in (8.1) by $I_{0}$, and the remaining integrals by

where

$$
\int_{\boldsymbol{R}^{n}} w(x) \prod_{i=1}^{r}\left(x^{i}\right)^{2 k_{i}} d V(\boldsymbol{x})=I_{2 k_{3}, 2 k_{2}, \ldots, 2 k_{r}}=\alpha_{r} \prod_{i=1}^{r} I_{2 k_{t}}
$$

$$
\sum_{i=1}^{r} 2 k_{i} \leqq 2 k, \quad k_{i} \geqq 1,
$$

$k_{i}$ being an integer. The integer $r$ will range from 1 to $k$.

Due to the full symmetry we need only consider those integrals (8.2) for which $k_{i} \leqq k_{j}$ if $i \leqq j, 1 \leqq i, j \leqq r$. The inequalities (8.3) tell us that for $v$ an integer in $1 \leqq v \leqq r$,

$$
2 \leqq 2 k_{p} \leqq 2 k-\min \sum_{i=1}^{\nu-1} 2 k_{i}-\min \sum_{i=p+1}^{r} 2 k_{i} .
$$

The maximum of the right hand side subject to the constraint $k_{i} \leqq k_{j}$ if $i \leqq j$ is obtained by setting $k_{i}=1$ for $i<v$ and $k_{i}=k_{\nu}$ for $i>\nu$. We thus find that $1 \leqq k_{v} \leqq k_{v}^{*}$, where

$$
k_{v}^{*}=k_{\nu}^{*}(r)=\left[\frac{k-\nu+1}{r-\nu+1}\right], \quad \nu=1,2, \ldots, r .
$$

Corresponding to the numbers $k_{\nu}^{*}$ we define numbers $l_{\nu}$ by

$$
l_{\nu}=l_{v}(r)=\min \left(\left[\frac{k+1}{2}\right], k_{*}^{*}(r)\right)
$$


We then list all the monomial integrals (8.2) subject to the constraints (8.3) together with the constraints

$$
k_{y} \leqq l_{v}, \quad y=1,2, \ldots, r
$$

where $l_{\nu}$ is defined by (8.6).

Let there be $x=\varkappa(r)$ distinct monomial integrals of the type (8.2) subject to the constraints (8.3) and (8.7). We shall write down the non-linear equations for a "minimum point" fully symmetric integration formula which exactly integrates these monomials, $r=0,1,2, \ldots, k$. Later we will show that this integration formula exactly integrates all the monomials (8.2) subject only to the constraint (8.3).

The $\left[\frac{k+1}{2}\right]$ distinct positive zeros $u_{1}, u_{2}, \ldots, u_{\omega}\left(\omega=\left[\frac{k+1}{2}\right]\right)$ of $q_{k+1}(x)$ will be used to construct a suitable set of $x(r)$ generators subject to the rules $(f)$ and $(\mathrm{g})$ of Section 3, such that we obtain a system of $\boldsymbol{x}(\boldsymbol{r})$ linear equations of a form similar to $(7.11)$ in the $x(r)$ unknown coefficients $A_{j_{1}}, j_{2}, \ldots, i_{r}$, for which the determinant does not vanish. Consider the system of equations

$$
\begin{array}{r}
\sum_{i_{1}=1}^{l_{r}(r)} \sum_{i_{1}=1}^{l_{r}(r)} \ldots \sum_{i_{r}=1}^{l_{r}(r)} w_{i_{1}} w_{i_{2}} \ldots w_{i_{r}} u_{i_{1}}^{2 k_{1}} u_{i_{2}}^{2 k_{k_{2}}} \ldots u_{i_{r}}^{2 k_{r}}=\alpha_{r} I_{2 k_{1}} I_{2 k_{2}} \ldots I_{2 k_{r}} \\
\left(k_{i}=1,2, \ldots, l_{r}(r) ; i=1,2, \ldots, r\right) .
\end{array}
$$

By the analysis of Section 7 we know that we may write (8.8) in the form of a system $\alpha$ of $\left(l_{r}(r)+r-1\right)_{(r)}$ linear equations which uniquely determines all of the $\left(l_{r}(r)+r-1\right)_{(r)}$ products $w_{i_{1}} w_{i_{2}} \ldots w_{i_{r}}, 1 \leqq i_{1} \leqq i_{2} \leqq \cdots \leqq i_{r} \leqq l_{r}(r)$. The $x(r)$ monomial integrals (8.2) subject to (8.3) and (8.7) are clearly a subset of those on the right of (8.8). Let the $x(r)$ monomials (labelled $1,2, \ldots, x(r)$ ) specified by (3.6) with $y=r$ together with (8.7), be used to yield the first $\psi(r)$ equations of the system $\alpha$. These $x(r)$ equations are clearly of rank $x(r)$, since the system $\alpha$ has a non-zero determinant. Thus, there exist at least $x(r)$ generators (labelled $1,2, \ldots, x(r))$ among the total $\left(l_{r}(r)+r-1\right)_{(r)}$, such that the square matrix, whose $(i, j)$-th element is the $i$-th monomial summed over the points generated by the $j$-th generator, has a non-zero determinant. Let $\boldsymbol{V}_{*(r)}^{(r)}$ denote that particular $\varkappa(r) \times x(r)$ matrix with non-zero determinant which is constructed from any particular set of generators selected such that the total number of points generated by them is minimized. In this way we shall minimize the total number of points required in our final integration formula. (We expect that if we list the generators in increasing order of the number of points each generates, then the square matrix $\boldsymbol{V}$ whose $(i, j)$-th element is the $i$-th monomial described above summed over the points generated by the $j$-th generator always has a non-zero determinant. However, we have so far been unable to prove this.) In general there will be many different choices of generators leading to different integration formulas, all of which require the same number of points. Observe that in choosing $\boldsymbol{V}_{\varkappa(r)}^{(f)}$ we have applied the rule (f) of Section 3 to select generators with exactly $r$ positive coordinates, and that we have applied the rule $(g)$ of Section 3 to minimize the number of points generated by the set of generators making up $\boldsymbol{V}_{\kappa(r)}^{(*)}$.

Assume that all the square matrices $V_{x(n)}^{(n)}$ have been determined as described above, for $r=1,2, \ldots, k$. We then also define the $x(r) \times \varkappa(s)$ matrices $V_{x(s)}^{(r)} s=$ 
$1,2, \ldots, k$ as follows. The $(i, j)$-th element of $\boldsymbol{V}_{*(s)}^{(r)}$ is the $i$-th monomial used to obtain $\boldsymbol{V}_{*(r)}^{(\eta)}$ summed over the points generated by the $j$-th generator in $\boldsymbol{V}_{x(s)}^{(s)}$. Here the Eq. (3.8) can be used to explicitly express each $(i, j)$-th element. Clearly $\boldsymbol{V}_{*(s)}^{(t)}$ is a $*(r) \times *(s)$ zero matrix if $r>s$.

Further we denote $\boldsymbol{W}^{(r)}(r=1,2, \ldots, k)$ to be a $x(r) \times 1$ dimensional vector of elements $A_{j_{1}, i_{3}, \ldots, i_{r}}$, the $j$-th element in $\boldsymbol{W}^{(r)}$ corresponding to the $j i t h$ generator used to construct $\boldsymbol{V}_{*(r)}^{(r)}$, and finally, we denote $I^{(v)}$ to be the $x(r) \times 1$ vector of monomial integrals, the $i$-th element of $I^{(r)}$ being the integral of the $i$-th monomial used to construct $\boldsymbol{V}_{*(r)}^{(r)}$.

At this point we are ready to consider solving the following sequence of $k$ systems of linear algebraic equations

$$
\begin{aligned}
& \boldsymbol{V}_{\varkappa(k)}^{(k)} \boldsymbol{W}^{(k)}=\boldsymbol{I}^{(k)} \\
& \boldsymbol{V}_{\varkappa(r)}^{(r)} \boldsymbol{W}^{(r)}=\boldsymbol{I}^{(r)}-\sum_{s=r+1}^{k} \boldsymbol{V}_{\varkappa(s)}^{(r)} \boldsymbol{W}^{(s)} \quad(r=k-1, k-2, \ldots, 1) .
\end{aligned}
$$

Clearly $\boldsymbol{W}^{(k)}$ is uniquely determined in (8.9). By induction it follows that each $\boldsymbol{W}^{(r)}(r=1,2, \ldots, k)$ in $(8,9)$ is uniquely determined, since the square matrix $\boldsymbol{V}_{*(r)}^{(r)}$ is non-singular.

This completes the proof that "minimum-point" generators can be found, and the corresponding coefficients $A_{j_{1}, j_{2}, \ldots, j_{4}}(s=1,2, \ldots, k)$ in $(8.1)$ can be uniquely determined such that the formula (8.1) is exact for all monomials of the form (3.6) (with $\nu=1,2, \ldots, k$ ), subject also to the constraint (8.7). Clearly, if we substitute $f=1$ into (8.1) we can now also determine $A_{0}$. Thus (8.1) is completely determined. Each coefficient $A_{0}, A_{j_{1}, j_{2}, \ldots, j_{s}}$ in $(8.1)$ is obviously real.

The right side of (8.1) gives us the number of points $m$ required by this formula if we substitute $A_{0}=A_{j_{1}, i_{2}, \ldots, j_{s}}=f=1$. By (3.4) this number of points is a polynomial in $n$, the number of dimensions. Since the total number of generators at the $s<k$ stage is independent of $n$, and since there is only one generator (with $k$ positive coordinates, all of which are the same) when $s=k$, it follows from (3.4) that the $s=k$ generator generates the dominant term in this polynomial. Hence

$$
m=\frac{(2 n)^{k}}{k !}\left[1+O\left(\frac{1}{n}\right)\right]
$$

It remains to show that (8.1) is exact for all the monomials (3.6) for $v=$ $0,1, \ldots, k$ without the additional restriction (8.7). Due to the full symmetry, we need merely carry out a proof for all those monomials subject also to (8.7) but with $l_{v}$ in (8.7) replaced by $k_{v}^{*}$ in (8.5). An easy calculation shows that $k_{v}^{*}(r)$ can be greater than $\left[\frac{k+1}{2}\right]$ only for $r=1,2, \ldots,\left[\frac{k}{2}\right]$, and also, subject to (8.7) with $l_{v}$ replaced by $k_{v}^{*}(r), k_{v}^{*}(r) \leqq\left[\frac{k+1}{2}\right]$ if $\nu<r$.

Let us fix an arbitrary integer $r$ in $1 \leqq r \leqq\left\lfloor\frac{k}{2}\right]$, and let $k_{v}^{*}=k_{v}^{*}(r)>\left[\frac{k+1}{2}\right]$. Substituting any monomial $f\left(x^{1}, x^{2}, \ldots, x^{*}\right)$ of the form $(3.6)$ for $y=r$ into $(8.1)$ we obtain

$$
\sum_{s=y}^{k} \sum_{1 \leqq j_{1} \leqq j_{1} \leqq \ldots \leqq j_{0}} A_{j_{1}, j_{k}, \ldots, j_{2}} \sum f\left[u_{j_{1}}, u_{j_{k}}, \ldots, u_{j_{r}}\right]=\alpha_{r} \prod_{i=1}^{r} I_{z_{k} k_{s}} .
$$


Dividing both sides of (8.10) by $\alpha, \prod_{i=1}^{r-1} I_{2 k_{i}}$ and on the left collecting coefficients of coordinates whose exponent is $2 k_{y}$, we obtain the equation

$$
\sum_{\mu} w_{\mu} u_{\mu}^{2 k_{r}}=I_{2 k_{r}}
$$

where the coefficients $w_{\mu}$ are functions of $k_{t}, t=1,2, \ldots, \gamma-1$. Let these integers $k_{t}$ be fixed subject to (8.3) and (8.7) with $l_{\nu}$ replaced by $k_{v}^{*}$, so that the integer $k_{r}$ in (8.11) is free to traverse over the range $1 \leqq k_{r} \leqq \omega$ where $\left[\frac{k+1}{2}\right] \leqq \omega \leqq k_{r}^{*}(r) \leqq k$.

For $k_{r}=1,2, \ldots,\left[\frac{k+1}{2}\right]$ the system (8.11) may be regarded as a system of $\left[\frac{k+1}{2}\right]$ linear equations in the unknowns $w_{\mu}$. Clearly the integer $\mu$ in (8.11) must range over all the integers in $1 \leqq \mu \leqq\left[\frac{k+1}{2}\right]$, for otherwise no solution to this system would exist. Thus the determinant of this system of $\left[\frac{k+1}{2}\right]$ equations is not zero, and so there exists a unique set of $\left[\frac{k+1}{2}\right] w_{\mu}$ 's such that (8.11) is exact for $k_{r}=1,2, \ldots,\left[\frac{k+1}{2}\right]$. However, by the definition of the $w_{\mu}$ in Section 5 and by the connection between orthogonal polynomials and Gaussian quadrature, it follows that once we have "determined" the $w_{\mu}$ by solving the system of $\left[\frac{k+1}{2}\right]$ linear equations, (8.11) is exact for $k_{y}=1,2, \ldots, k$.

This completes the proof of Theorem 8.1 .

To facilitate the evaluation of the right of (8.1) we suggest using the formula

$$
\sum t\left[u_{i_{1}}, u_{j_{2}}, \ldots, u_{j_{s}}\right]=\sum_{i_{1}=-p}^{p} \sum_{i_{2}=-p}^{p} \ldots \sum_{i_{n}=-p}^{p} \delta\left(i_{1}, i_{2}, \ldots, i_{n}\right) f\left(u_{i_{1}}, u_{i_{2}}, \ldots, u_{i_{n}}\right) .
$$

In (8.12) $u_{-j}=-u_{j}$ for $j>0, u_{0}=0$, and $\delta\left(i_{1}, i_{2}, \ldots, i_{n}\right)$ is defined as follows. It is presumed that the generator $\left[u_{j_{1}}, u_{j_{2}}, \ldots, u_{j_{s}}\right]$ has $p$ distinct positive coordinates: $p_{1}$ of the first, $\ldots, p_{p}$ of the $p$-th so that $\sum_{i=1}^{p} p_{i}=s$. Put $G^{*}=\left\{j_{1}, j_{2}, \ldots, j_{s}\right.$, $\left.j_{s+1}, \ldots, j_{n}\right\}$ where the integers $j_{1}$ to $j_{s}$ are defined as for $(8.12)$, and $j_{s+1}=j_{s+2}=$ $\cdots=j_{n}=0$. Then $\delta\left(i_{1}, i_{2}, \ldots, i_{n}\right)$ is respectively equal to one or zero according to whether or not the set $\left\{\left|i_{1}\right|,\left|i_{2}\right|, \ldots,\left|i_{n}\right|\right\}$ is a permutation of the elements in $G^{*}$. Accordingly, it is unnecessary to compute $f\left(u_{i_{2}}, u_{i_{2}}, \ldots, u_{i_{n}}\right)$ whenever $\delta\left(i_{1}, i_{2}, \ldots, i_{n}\right)=0$.

Although the above constructive proof is carried out only for $n \geqq k$, it applies equally for $n<k$ provided we define $\boldsymbol{I}^{(r)}=\boldsymbol{O}$ for $r>n$.

\section{Conclusion}

At the outset of the paper we have given a simple transformation which transforms integrals over a large class of regions into integrals over the $n$-cube.

In the remainder of the paper we have concentrated on the development of a procedure for constructing fully symmetric numerical integration formulas. To start with, we have obtained four fully symmetric formulas of degree 3, 5, 7 and 9 by directly solving a system of non-linear algebraic equations. Difficulties inherent in these special cases are later overcome in the general degree $2 k+1$ case by restricting ourselves to integrals with property $\boldsymbol{P}$ (Section 6 ), by introducing 
orthogonal polynomials (Section 5), generators (Section 3), by establishing a connection between repeated integration and generators (Section 7) and by using the connection between Gaussian integration and the solution of a certain corresponding system of non-linear algebraic equations. Thus the problem of solving the system of non-linear algebraic equations in the general degree $2 k+1$ case is reduced to solving a sequence of $k+1$ systems of linear algebraic equations.

It is apparent from tabulation of the formulas for moderate or large $n$ and $k$ that the weights of the minimum-point formulas of the preceeding section differ in relative magnitudes by as much as $10^{5}$ for e.g. $n=5,2 k+1=7$.

It seems that it may be difficult to find formulas which satisfy all the criteria mentioned in the introduction of this paper. In Section 3 the positive weight formula was rejected because the coordinates of the evaluation points for it are not zeros of certain orthogonal polynomials. Moreover, these evaluation points are in many cases outside the region of integration when $n$ is large. Abandonment of the positive weight criterion may lead to formulas involving large weights or too many evaluation points. A possibility which we have not examined is to use linear combinations of the formulas of the present paper in such a way as to minimize the weights.

\section{References}

[1] Thacher, H. C. JR.: Optimum quadrature formulas in $s$ dimensions. Math. Comput. V. 2, $69-73(1957)$.

[2] Hammer, P.C., and A. W. Wymore: Numerical evaluation of multiple integrals I. Math. Comput., V. 2, 59-67 (1957).

[3] Hammer, P. C., and A. H. Stroud: Numerical evaluation of multiple integrals II. Math. Comput. V. 12, 272-280 (1958).

[4] Stroud, A. H.: Approximate integration formulas for certain spherically symmetric regions. Math. Comput. V. 17, 105-135 (1963).

[5] LynEss, J. N.: Symmetric integration rules for hypercubes I. Error coefficients. Math. Comput. V. 19, 260-276 (1965).

[6] Lyness, J. N.: Symmetric integration rules for hypercubes II. Rule projection. and rule extension. Math. Comput. V. 19, 394-407 (1965).

[7] LyNESS, J. N.: Symmetric integration rules for hypercubes III. Construction of integration rules using null rules. Math. Comput. V, 19, 625-637 (1965).

[8] Stenger, F.: Numerical integration in $n$ dimensions. Thesis, University of Alberta (1963).

[9] Tchakaloff, V.: Formulas de cubature mecanique à coefficients non-negatif. Bull. Sci. Math. 81 (2), 123-134 (1957).

[10] Stenger, F.: Error bounds for the evaluation of integrals by repeated Gausstype formulae. Numer. Math. 9, 200-213 (1966).

Canadian Mathematical Congress

985 Sherbrooke St., West

Montreal, Canada
Dept. of Mathematics

University of Michigan

Ann Arbor, Michigan 48104, USA 